\title{
Mobile Claims Assistance
}

\author{
Oliver Baecker* \\ Institute of Technology \\ Management \\ University of St. Gallen \\ oliver.baecker@unisg.ch
}

\author{
Tobias Ippisch \\ Institute of Technology \\ Management \\ University of St. Gallen \\ tobias.ippisch@unisg.ch
}

\author{
Florian Michahelles \\ Department of Management, \\ Technology, and Economics \\ ETH Zurich \\ fmichahelles@ethz.ch
}

\author{
Sascha Roth \\ SAP Research \\ Switzerland \\ SAP AG \\ sascha.roth@sap.com
}

\author{
Elgar Fleisch ${ }^{\ddagger}$ \\ Institute of Technology \\ Management \\ University of St. Gallen \\ elgar.fleisch@unisg.ch
}

\begin{abstract}
When it comes to vehicle accidents, people are stressed out and overstrained, even if it is just a car body damage and no one is hurt. They often lack adequate and immediate assistance and may worry about the lengthy and paper-based loss report to their insurance carrier. At the same time, it is crucial for insurance companies to receive early and detailed case circumstances in order to decrease costs and assist customers with value-added services. Against this background, we propose the usage of mobile phones in order to assist people in the aftermath of an accident. We present a concept for mobile claims assistance along with a prototypical implementation that features an asynchronous communication between mobile phones and claims management enterprise systems based on mobile Web Services. Finally, we discuss the user perspective on mobile insurance applications and present data we collected using a combination of focus groups and user surveys.
\end{abstract}

\section{Keywords}

Mobile Computing, Mobile Web Services, Prototype, Assistive Technologies, Emergency Response, Claims Management

\section{INTRODUCTION}

Minor car accidents are unpleasant and emotionally stressful events. Often people lack adequate assistance in terms of immediate services like the arrangement of a tow truck or support with the loss report to their insurance company. When insurance global player Zurich questioned 39,000 of its customers what they were looking for in case of an insurance claim such as a car accident, the answer was:"Help that shortens the distance between problems

\footnotetext{
* Oliver Baecker also works with SAP Research Switzerland

${ }^{\ddagger}$ Elgar Fleisch also works with D-MTEC, ETH Zurich
}

Permission to make digital or hard copies of all or part of this work for personal or classroom use is granted without fee provided that copies are not made or distributed for profit or commercial advantage and that copies bear this notice and the full citation on the first page. To copy otherwise, or republish, to post on servers or to redistribute to lists, requires prior specific permission and/or a fee.

MUM09, November 22-25, 2009 Cambridge, UK.

Copyright (c) 2009 ACM 978-1-60558-846-9 09/11 \$10.00 and resolution.”1 In order to get immediate help and benefit from adequate service offerings, there needs to be a way to communicate this urgent desire. While the direct contact to a human counterpart is the normal case in this situation, the mobile phone has emerged as the most dominant mediation device in safety-critical situations: qualitative interview data as well as quantitative analysis show that people's sense about mobile telephony is to never be alone in emergencies [15]. Accordingly, we see an important role for mobile phones in emergencies such as car accidents. In addition, our decision to investigate the application of mobile phones in the context of claims assistance is based on seven focus groups and a previous user survey, which we conducted as part of a broader research stream on the role of mobile technology in the insurance domain (details are provided in section 5).

The outlined customer perspective is complemented by the business perspective where insurance companies suffer from problems in terms of data quality, media breaks, and delayed loss reports, which lead to both high operational costs and loss expenses. As of today, insurance companies use dedicated enterprise systems to manage the overall claims management business process. However, available claims management systems are poorly, if at all, integrated with the physical world of insured objects and persons they are supposed to manage. This means, insurance claims are reported via traditional channels such as phone, letter, or (in the best case) online portals and are then keyed in by insurance personnel. As recent studies indicate, cost savings based on an earlier loss report and a pro-active claims management can reach up to $15 \%$ [8], which equals a double-digit million dollar saving per year for a mid-size insurance company. Consequently, insurers as well as technology providers recently launched mobile claims assistance applications to unlock the cost saving potential [4] [18]. However, state-of-the-art solutions lack a direct and asynchronous integration between mobile phone applications and claims management enterprise systems. Instead, existing approaches involving mobile phones are based on e-mail communication or text messages as opposed to native mobile phone applications.

\footnotetext{
${ }^{1}$ http://www.zurich.com/main/about/helppointprinciples/introduc tion.htm
} 
Against this background, we present a mobile claims assistance application as well as an integration architecture that features the communication between mobile phones and claims management enterprise systems. The application enables an emergency call in combination with a loss report from the mobile phone to a business application, provides location-based services to the customer, and allows insurance companies to request customer feedback after the claim is settled. To realize the outlined services, the architecture enables direct and asynchronous communication between mobile phones and claims management business applications.

The contribution of this paper is the development of an innovative mobile application for a novel application domain together with the proposal of required enabling technologies. Based on the concepts for mobile claims assistance described in section 3 , the practical contribution of this paper is a prototypical implementation that shows how mobile phones can be integrated with claims management enterprise systems in order to assist people after car accidents. As outlined in section 4, the technical contribution of this work is the development of the required enabling technologies such as the integration architecture that allows for a direct and asynchronous communication between mobile phones and business applications based on mobile Web Services. In section 5, we discuss the user perspective on mobile insurance applications based on data we collected using a combination of focus groups and user surveys. The paper concludes with a discussion of our main findings and an outlook on future work.

\section{RELATED WORK}

With the emergence of ubiquitous computing devices, risk-, health-, and safety-related data is being captured at an increasingly detailed level and provided to users via mobile devices. Besides informing users via text messages, mobile devices are used as interfaces for tasks like supporting the user's daily routine with selective risk information [14], providing avalanche warnings for off-piste skiing [23], and managing ad-hoc assistance teams [1]. The provision of such risk-related information can be offered by insurance companies or travel agencies as value-added services. While the aforementioned applications focus on proactively providing risk-related information to users, our application aims at assisting users after the occurrence of a loss by means of locationbased services.

The usage of mobile phones for emergency response work is discussed in [13]. The authors focus on the role of mobile phones for the initial interaction between involved persons after major incidents. The work identifies different usage patterns for mobile devices and underpins the emerging importance of mobile phones in assisting people during emergency response activities.

Early business applications of ubiquitous computing technology targeted areas such as supply chain management, or industrial monitoring. However, the application of ubiquitous computing technology in the insurance industry gains in importance [3]. Based on Car-2-X technology [7], motor vehicles get connected to insurance enterprise systems, which enables usage-based pricing models [16], allows for emergency calls [6], and facilitates recording of crash data [21]. While the outlined applications are currently deployed in motor vehicles, similar applications will become available for mobile phones as their computational power increases, additional sensor technology becomes available, and mobile phones get integrated with enterprise systems. As an ex- ample, the iPhone App Store offers two different claims assistance applications, "Crash Report" [4] and "C.A.R. - Car Accident Report” [10], which support users with a loss report from the iPhone. Our prototype differs from both applications as it is integrated with a commercial claims management enterprise system and provides location-based services instead of simply capturing and transmitting data via e-mail.

\section{CONCEPT}

The concept of the proposed mobile claims assistance application is based on typical activities in the aftermath of a car accident. While the different processes are well-described in literature [8], we also conducted expert interviews to take account for the special role of insurance companies. The overall process can be separated into three phases:

\section{An emergency call / loss report from the mobile phone, \\ 2. subsequent location-based services that are offered on the device, and}

3. customer feedback after the insurance claim is settled.

\subsection{Emergency Call}

In the first phase, the mobile claims assistance application is used to submit an emergency call together with a loss report to an insurance company. As shown in Figure 1 a), the application can be used to directly place the emergency call, which establishes a voice connection to an emergency call center and simultaneously submits the current position as well as personal data about the policy holder. If there is no need for an immediate emergency call, one can add additional information before submitting the loss report. As an example, incident types such as "collision", "breakdown", "breakage of glass", or "theft" can be specified. Furthermore, the application can be used to enrich the loss report with supplementary information like pictures of the accident scene or data about the other party. As shown in Figure $1 \mathrm{~b}$ ), the opponent's personal data can be entered manually or by scanning a two-dimensional barcode from an insurance card using the mobile phone camera [9]. The outlined additional information can be either collected before submitting the claim, while talking to an insurance agent that guides the customer through the process, or later on as a supplement to the initial loss report. A loss report containing information about the policy holder, the loss location, the other party, and images of the accident scene is shown in Figure 2 a).

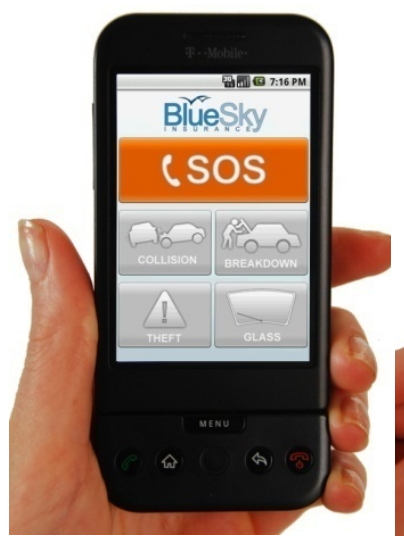

a)

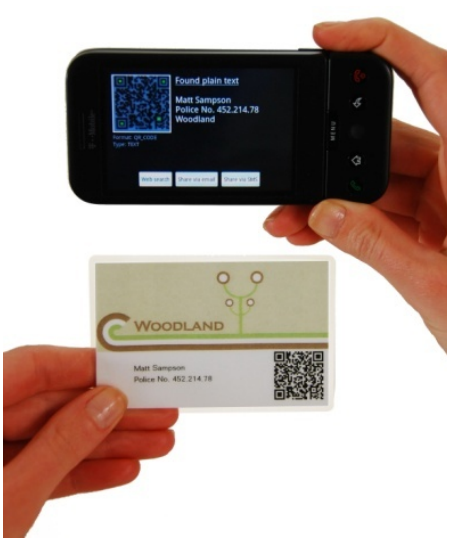

b)
Figure 1. a) Emergency Call b) Barcode Scan 


\subsection{Location-Based Services}

In a second phase, the mobile phone application is used to assist users with different location-based services. The current location is either determined via a built-in GPS module or, as a fallback, based on the GSM cell the phone is using. The place of accident is an especially time-critical piece of information, as it enables the instantaneous coordination of emergency services as well as the provision of value-added services on the mobile phone. As shown in Figure 2 b), the insurance company offers services to the customer right after the claim was submitted to the claims management enterprise system. As an example, the user can request a tow truck or, in case the car is still roadworthy, use a maps application to navigate to the closest authorized repair shop. The application also provides helpful information like a customer's eligibility for a rental car as well as the arrival time of a requested tow truck. The list of service providers is based on information stored in the enterprise system and takes into account the current GPS position of the mobile phone user as well as the location of suitable service providers.

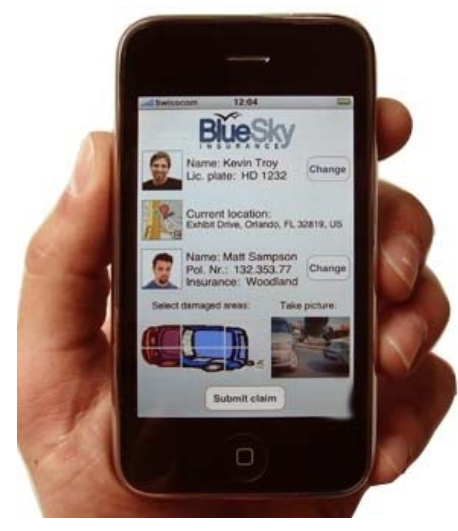

a)

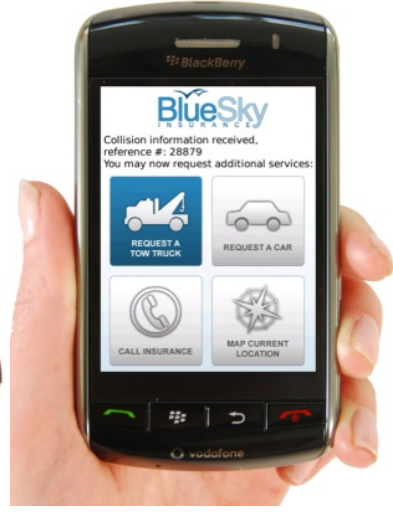

b)
Figure 2. a) Loss Report b) Location-Based Services

\subsection{Customer Feedback}

Finally, the transmitted information, e.g. pictures of the accident scene or the location of the accident, can be reviewed in the claims management enterprise system from the perspective of claim personnel at an insurance company. The claim file also contains information about the automatically assigned business partners that offer various third party services on the mobile phone. In order to improve their customer service, it is of high relevance for insurance companies to collect feedback about customers' satisfaction with the overall claims management process. Therefore, the mobile claims assistance application allows insurance companies to request customer feedback from within their claims management systems. That means, whenever a claim is closed in the enterprise application, a business rule is triggered and a survey notification (see Figure 3 a)) is sent to the mobile phone where the user can launch the customer feedback screen (technical details on how to invoke a mobile Web Service from a business application are discussed in sub-section 4.2). The content of the feedback screen (i.e. the feedback questions) is dynamically sent to the mobile phone when invoking the mobile Web Service.

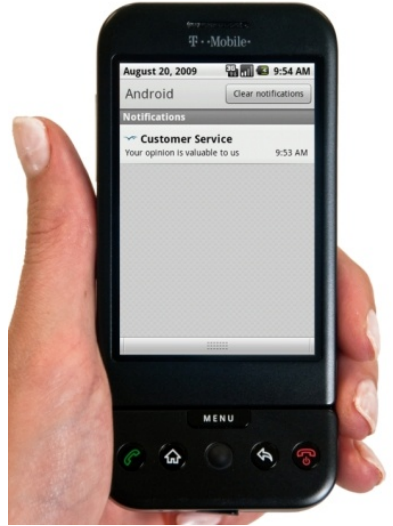

a)

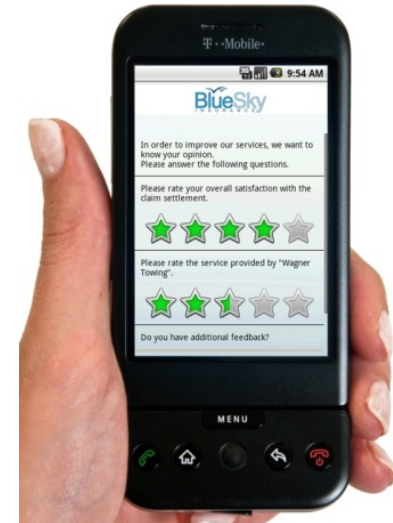

b)
Figure 3. a) Survey Notification b) Service Rating

As illustrated in Figure $3 \mathrm{~b}$ ), the screen consists of a combination of a text input field and star bars to enable the rating of the overall satisfaction with the claims management process as well as to provide additional feedback. In addition, the customer can rate services provided by business partners such as repair shops or tow truck companies. The aggregation of this data in combination with the location information provides the insurance carrier with a holistic view on the service quality provided by his business partners in different regions. The information can then be used to improve the overall service level for customers by cooperating with the appropriate business partners. In addition, the customer feedback can be used to improve offered services, thereby improving customer satisfaction and loyalty.

\section{PROTOTYPICAL IMPLEMENTATION}

For our prototypical implementation, we developed client applications for the Android platform (HTC's G1 mobile phone), BlackBerry OS (BlackBerry Storm 9500), and the iPhone 3G. The combination of an emergency call (see sub-section 3.1) and location-based services (see sub-section 3.2) is implemented on all three platforms, while the customer feedback (see sub-section 3.3) is so far available on the Android platform. The decision to develop native mobile phone applications instead of browser-based solutions enables direct access to mobile phone hardware modules. For instance, the application can access the build-in camera to scan a barcode in order to record the opponent's data or it can make use of the GPS module to offer location-based services. Finally, an integration architecture is used to connect the mobile phone to SAP's claims management solution "SAP Claims Management 6.0 EHP4"².

\subsection{Integration Architecture}

The integration architecture illustrated in Figure 4 connects mobile phones residing at the device layer with claims management enterprise systems residing at the business layer. It consists of a set of Enterprise Java Beans (EJB) 3.0 components, which offer their functionality as Web Services. The application of Web Service technology allows for an easy integration with business layer applications that increasingly expose enterprise functionality by means of Web Services (so-called "Enterprise Services") in the context of service-oriented architectures (SOA). By extending the Web Service paradigm to the device layer, we enable a Web

\footnotetext{
${ }^{2}$ http://www.sap.com/industries/insurance/claimsmanagement.epx
} 
Service-based communication with the integration architecture and thus with business applications. This approach also follows recent efforts to solve interoperability issues by using Web Services at the device layer [11], [22]. In particular, using SOAP (Simple Object Access Protocol) as a communication protocol dramatically reduces the development time when integrating mobile phones with business applications.

In order to provide for compatibility and ease of integration, the Event Handler component implements the OASIS Web Services Notification (WSN) standard [20]. This standard incorporates relevant functionality such as the publication of dedicated events to subscribers through a generic Web Service operation. The usage of a publish/subscribe mechanism is beneficial for several reasons. Firstly, it decouples mobile phones from enterprise systems, which is crucial when it comes to interface modifications in the enterprise system. Secondly, it reduces network traffic, because a mobile phone application publishes an information only once instead of sending it to every enterprise system separately. Thirdly, this approach increases the level of security, as mobile phones cannot access enterprise systems directly.

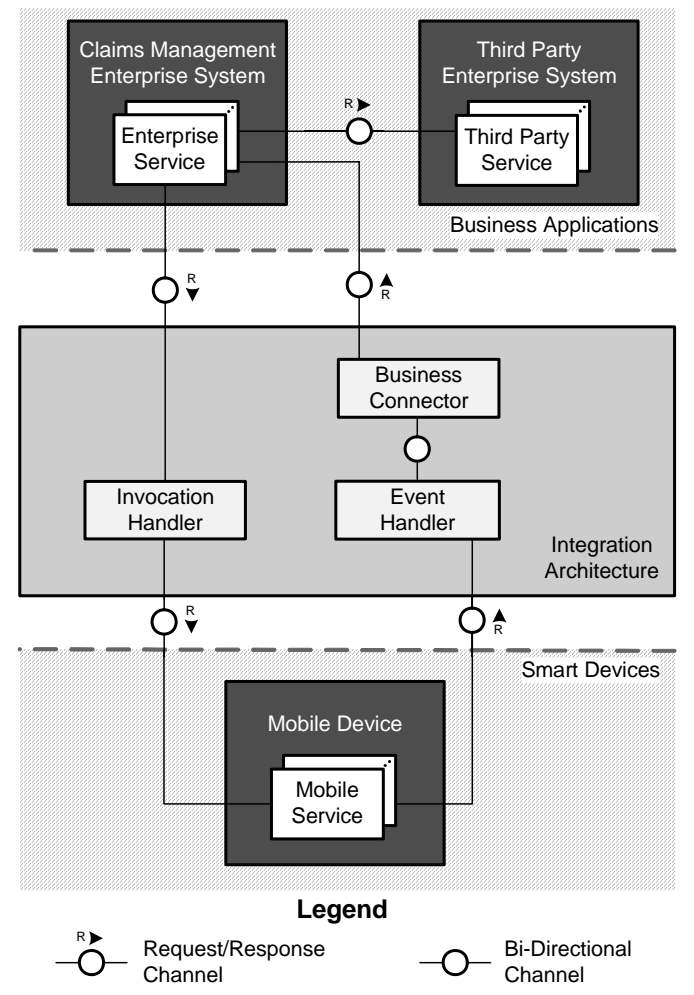

Figure 4. Integration Architecture

To implement the concepts outlined in section 3, the Event Handler component enables mobile phones to publish loss reports as events via Web Service interfaces. The Business Connector subscribes to these events and gets notified by the Event Handler. The component encapsulates the application logic needed for transforming device layer events to actions in business applications. In addition, it provides a layer of abstraction with respect to the business application deployed for a given scenario. This allows integrating additional enterprise applications by instantiating further business connectors. In addition, existing enterprise appli- cations can be modified without affecting the mobile phone client and the Event Handler component.

Figure 5 provides a detailed view on the role of the Invocation Handler component, which enables the invocation of mobile Web Services. To enable a service invocation from within a business application, the Tunnel Connector on the mobile device establishes a persistent TCP/IP connection to the Invocation Handler at system startup. This TCP/IP connection is used as a network tunnel in order to invoke mobile Web Services offered by the Web Service Container on the mobile device. Tunneling the network is necessary, because most mobile phone provider networks are using Network Address Translation (NAT) to minimize the allocation of public IPv4 addresses. As a consequence, there exists no public service endpoint on the mobile device when operating in a regular mobile phone provider network. The approach of using a minimized TCP tunneling enables us to use a previously established connection to invoke service endpoints on mobile devices without producing any network traffic overhead. Also, the endpoint of a WSDL that is queried through the Invocation Handler is transformed using a DOM parser to beware location transparency. Location transparency guarantees that, if the device is connected to the Internet, its Web Services can be invoked via a static endpoint, hiding all other infrastructure, such as the Tunnel Connector. Thus, the resulting WSDL file contains an endpoint address, which directly points to the Invocation Handler. Furthermore, the endpoint is enriched with the International Mobile Subscriber Identity (IMSI) of the mobile phone offering the respective service such that the Invocation Handler is able to identify the device. Finally, at the business layer, third party services are invoked from within the claims management enterprise system, for example, to enrich the loss report with weather data or to send a work order to a repair shop.

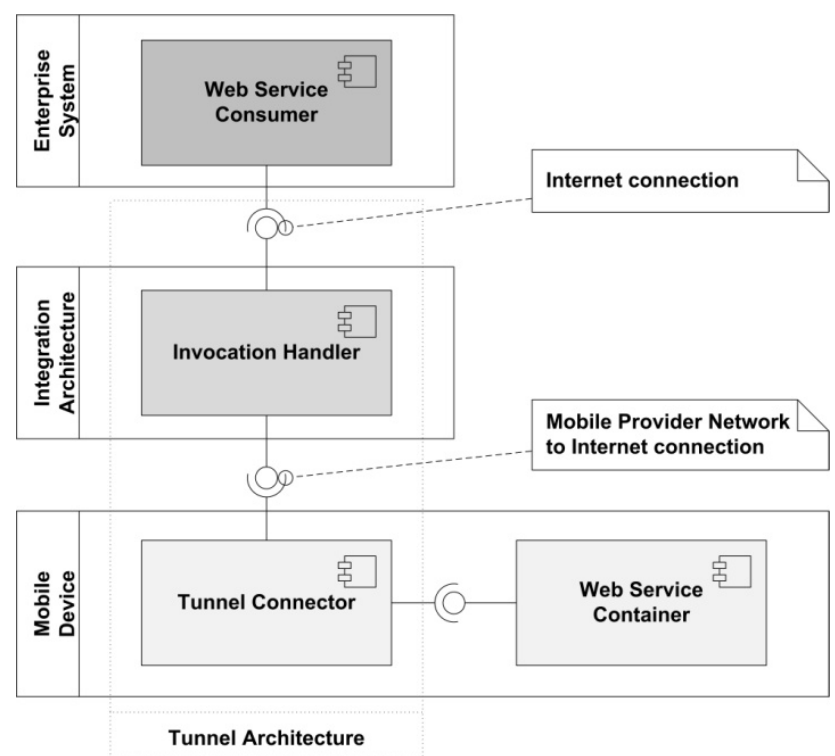

Figure 5. TCP Tunnel Architecture

\subsection{Mobile Web Services}

To actually provide Web Services on a mobile device, we developed a Web Service Container for the Android platform. Since Android is not fully Java-compliant, but supports parts of the Java core libraries, the Java reflection API is used to dynamically load 
platform-dependent classes and execute their methods. Also, the reflection API is used to implement a hot-deployment mechanism to add Web Services to the container. When developing a library for the Android platform, the missing Java-compliancy becomes an issue, e.g. a String. isEmpty ( ) method causes the application to crash at runtime. Furthermore, there are no libraries available for providing Web Services on the Android platform. Thus, we started from scratch using ordinary Java Sockets and a SAX parser to implement the marshaling for SOAP messages. In addition, Android influences the build process, i.e. .class files are not sufficient for running inside the VM of Android. Hence, we use a multiplatform . jar file for deploying new Web Services, which contains .class files and a . dex file that runs on the Android VM. As a result, our multiplatform . jar file also runs on ordinary Java VMs, which becomes beneficial when migrating our mobile Web Service implementation to further Java-based mobile phone platforms.

Approaches similar to the use of mobile Web Services were recently introduced to the market. As an example, Apple launched the Push Notification solution for the iPhone OS 3.0 [21]. Similar to the approach introduced in this paper, this solution keeps a persistent connection to the phone to be able to push new notifications to the device and launch applications remotely. In comparison to Apple's solution, our approach goes beyond a push-based notification of mobile applications. Especially when it comes to enterprise applications, it enables an easy integration of mobile devices into existing business processes using WS-* standards like the business process execution language (BPEL). In addition, Apple's Push Notification solution is proprietary, while our solution could be easily migrated to other mobile phone platforms.

After the proposal of a concept for mobile claims assistance and the presentation of our prototypical implementation, the following section 5 provides a user perspective on mobile insurance. This section complements our technical contribution by constituting our research focus and by evaluating the mobile claims assistance application.

\section{THE USER PERSPECTIVE}

As outlined in the introduction, our decision to investigate the application of mobile phones in the context of claims assistance was based on seven focus groups and a user survey. Both studies were conducted as part of a broader research stream on the role of mobile technology in the insurance domain. The initial studies were then complemented by a second user survey that evaluated the mobile claims assistance application together with four other mobile insurance applications in greater detail.

\subsection{Goals, Set-up, and Procedure}

The goal of the combination of focus groups and surveys was to examine, which mobile insurance applications are of interest for end users. In addition, we wanted to examine how users rate the identified applications in several dimensions, such as their perceived usefulness and users' intention to use the applications. An initial list of 21 mobile insurance applications was identified based on seven focus groups (see sub-section 5.2), and a first survey with a smaller sample of 300 participants was used to identify the five best-rated applications (see sub-section 5.3). Based on a larger sample of 2,000 participants, we then evaluated the five best-rated applications in greater detail (see sub-section 5.4 and 5.5).

\subsection{Focus Groups}

Over a period of two months, we organized a total of seven focus groups together with a large telecommunications provider as part of a joint research project on mobile insurance applications. Each focus group was joined by representatives of a different insurance company (i.e. one insurance company per focus group), by differing end users (represented by students), and members of the research team. All workshops started with outlining recent developments in mobile technology and showing live demos of mobile applications in the insurance context to stimulate discussion. During the workshops, the participants pinpointed insurance challenges that can be addressed by mobile phone applications and sketched first corresponding application scenarios. Based on the seven focus groups, we collected various ideas for mobile insurance applications and consolidated them to an initial list of 21 possible applications (see Table 1 ).

\section{Table 1. Mobile Insurance Applications}

1. Adjustment of existing insurance coverage

2. Situation-based insurance coverage

3. Mobile access to the extent of insurance coverage

4. Mobile-based insurance sales

5. Health-supporting shopping assistant

6. Individualized product information

7. Mobile-based health communities

8. Mobile-based policy / contract manager

9. Premium-saving portal

10. Location-based weather information

\subsection{User Survey I: Application Ranking}

In order to focus our research on selected mobile insurance applications, we conducted an initial user survey. The sample of 300 participants was drawn from a commercial online panel and was controlled for gender and age in order to ensure representativeness in these dimensions. Our goal was to get an assessment of the 21 applications by potential users regarding their perceived market potential. The participants of the web-based survey were asked to answer the question: "How do you estimate the market potential of the following applications?" The participants could assess the individual applications using sliders that allowed values from $0 \%$ (no market potential) to $100 \%$ (high market potential). The five best-rated applications (and their average ratings) were: 
1. Emergency button (81\%)

2. Mobile first aid guide (58\%)

3. Location-based weather information (55\%)

4. Mobile property monitoring (48\%)

5. Mobile first notice of loss (48\%)

Based on the results of the combination of focus groups and our first user survey, we started to develop the mobile claims assistance prototype presented in section 3 that incorporates the functionalities of the applications "emergency button" and "mobile first notice of loss". We favored those two applications over the others, as they impose the challenge of establishing an asynchronous communication channel between mobile phones and enterprise systems (as opposed to "location-based weather information”), because they require less investments in infrastructure (as opposed to "mobile property monitoring"), and since they go beyond a collection of illustrative screens requiring more complex human-machine interaction (as opposed to "mobile first aid guide”).

\subsection{User Survey II: Application Assessment}

In parallel to the development of the prototype, we analyzed the perception of the five best-rated applications in greater detail in a second evaluation through a web-based empirical survey amongst 2,000 participants. Again, the sample was drawn from a commercial online panel and was controlled for gender and age in order to ensure representativeness in these dimensions. At the outset, each user had to provide demographic information. Next, consumer attributes on mobile phone use, innovativeness, insurance involvement and risk attitude were queried. In a third part, the single applications were evaluated in detail. Owing to time considerations, only two out of the five applications were randomly assigned to the participants, which were given both a stimulus text and some mock-up screen shots to illustrate the functionality of each application. For the "mobile first notice of loss" application, the stimulus text was:

"Minor car damages (parking damages, rear-end collisions) or breakdowns can be reported to the insurance company via the mobile phone. Based on the current location, the application allows contacting the closest towing service or provides directions to the next body shop. In addition, the instant notification of the insurance company speeds up the claims management process. With the electronic claims notification being sufficient for claims handling in most cases, additional paperwork can be omitted."

Figure 6 shows two corresponding illustrations of the application that were provided to survey participants. For each application, the participants were asked to rate the applications' perceived usefulness, ease of use, credibility, and their attitude and intention to use towards the mobile insurance applications. In a final step, it was assessed whether such applications would foster word of mouth communication and impact consumers' willingness to pay more for insurance products that are combined with additional mobile applications. All items of the single constructs were administered on 7-point Likert scales ranging from 1 (strongly disagree) to 7 (strongly agree).
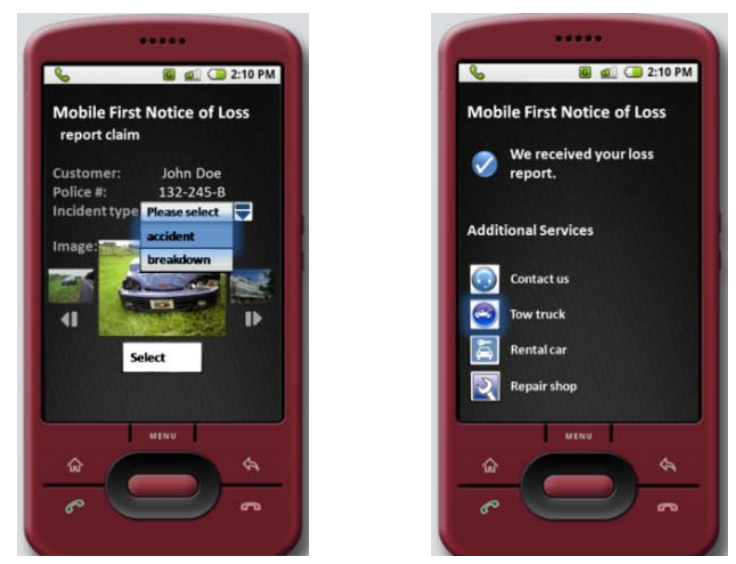

Figure 6. Mock-up Screens for User Survey II

\subsection{Selected Results}

In the following, selected results of the empirical analysis are presented. Figure 7 illustrates the results for the construct "usefulness" (represented by four items as derived from [5] and [19]) of the five applications. The figure shows the mean as well as the standard deviation (visualized as a black curve around the mean) for the four items per application. All ratings for "mobile first notice of loss" and "emergency button" are above the average value of 4 and the applications are regarded as especially useful in helping people to react quickly and properly in case of an emergency. Also overall usefulness for both applications, queried by the last sub-question, was answered significantly above average. Especially in the case of the "mobile first notice of loss" application, this actually was surprising to us, as we expected participants to be rather reluctant towards this application due to its novelty and the fact that it requires some more user engagement than the other tested applications.

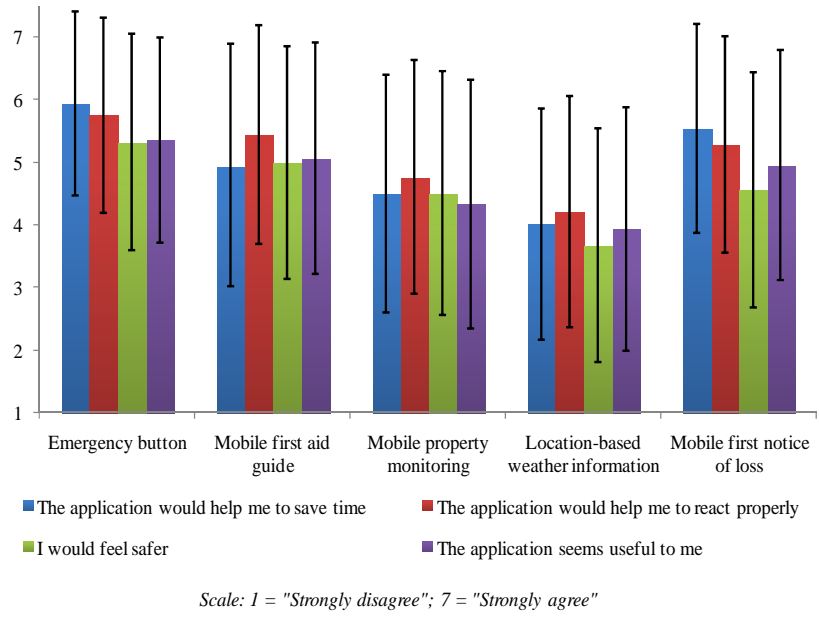

Figure 7. User Assessment (Construct “Usefulness”) 
Similar results, especially when comparing the different applications, are given in Figure 8, which illustrates the outcomes for the construct "intention to use" (represented by two items as derived from literature, cf. [5] and [19]).

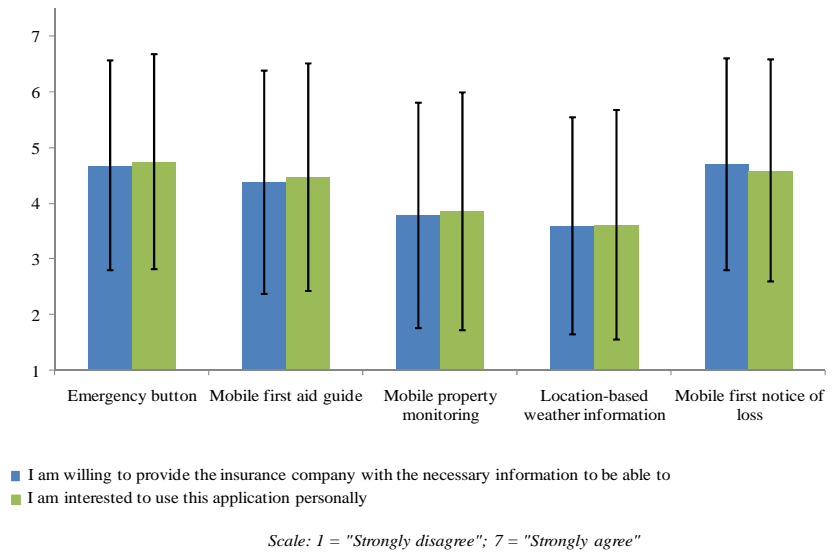

Figure 8. User Assessment (Construct “Intention to Use”)

When comparing the ratings, it is noticeable that applications, which provide assistance in concrete emergency situations (i.e. "emergency button", "mobile first notice of loss", and "mobile first aid guide") score significantly higher than those with a more preventive character (i.e. "location-based weather information" and "mobile property monitoring"). This shows evidence that people favor reactive assistance applications over proactive prevention applications.

As a next step, we performed a categorization of survey participants with respect to their mobile phone usage and their innovativeness. We pursued a principal component analysis [12] to identify three different types of mobile phone users as shown in Figure 9.

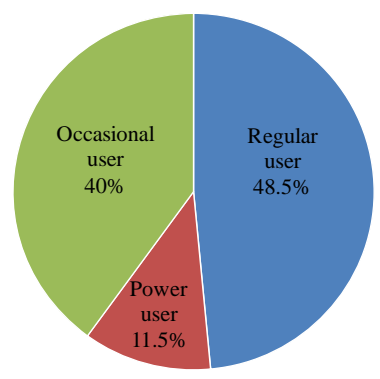

Figure 9. Mobile Phone User Types

The three user categories identified by our analysis were interpreted as occasional mobile phone users, regular users, and socalled "power users". The first group can be characterized by low monthly mobile phone expenses. It operates predominantly on prepaid plans and limits itself to using the mobile phone for calls and text messages. Correspondingly, the mobile phones used by members of this group exhibit rather limited functionality. Next, the group of regular users conveys medium monthly mobile phone expenses, often as part of a monthly mobile phone plan. Mobile devices come with solid functionality as they are usually replaced upon contract renewal. The last, smallest group consists of socalled "power users". Monthly mobile phone bills of members of this group are high; devices used are predominantly smart phones, such as RIM's Blackberry devices, Apple’s iPhone, HTC’s G1, or
Nokia's N Series devices. A look at the last group’s mobile usage pattern shows that it makes use of a broad range of mobile applications already today.
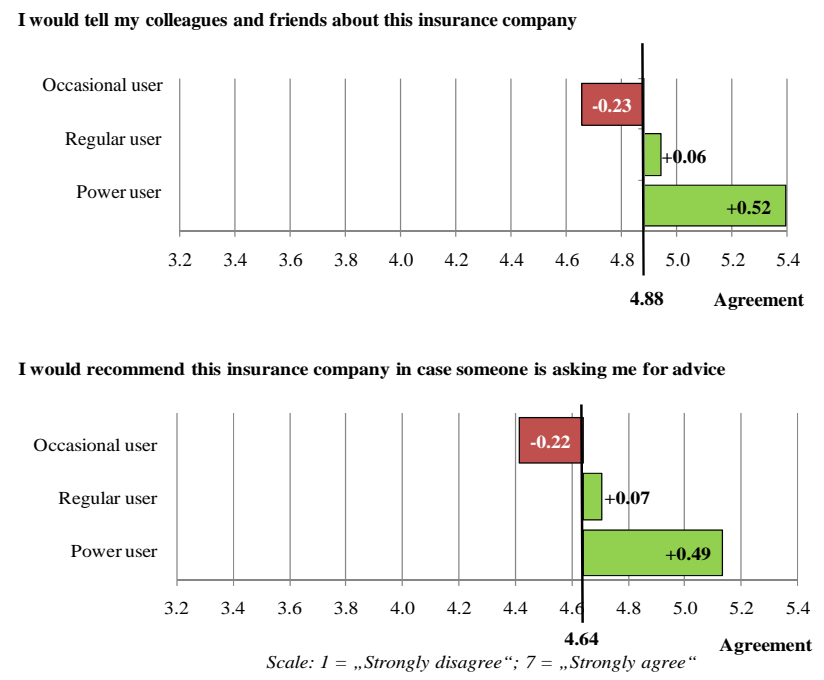

Figure 10. Word-of-Mouth (by User Type)

We now use this classification of survey participants to look into more details on differences in response behavior with respect to word-of-mouth advertisement and willingness-to-pay-more (this data has been queried on a per-user and not on an application level). For measuring any effects on word-of-mouth communication, two items were derived from [24] and adjusted to the mobile service context. Similarly, effects on the user's willingness to pay were measured by two items from [25]. As Figure 10 shows, "power users" are significantly different from the other user groups regarding their willingness to talk about or recommend insurance companies offering mobile services. We assume that this goes along with the "demonstration potential" of innovative mobile phone applications when talking to colleagues or friends.
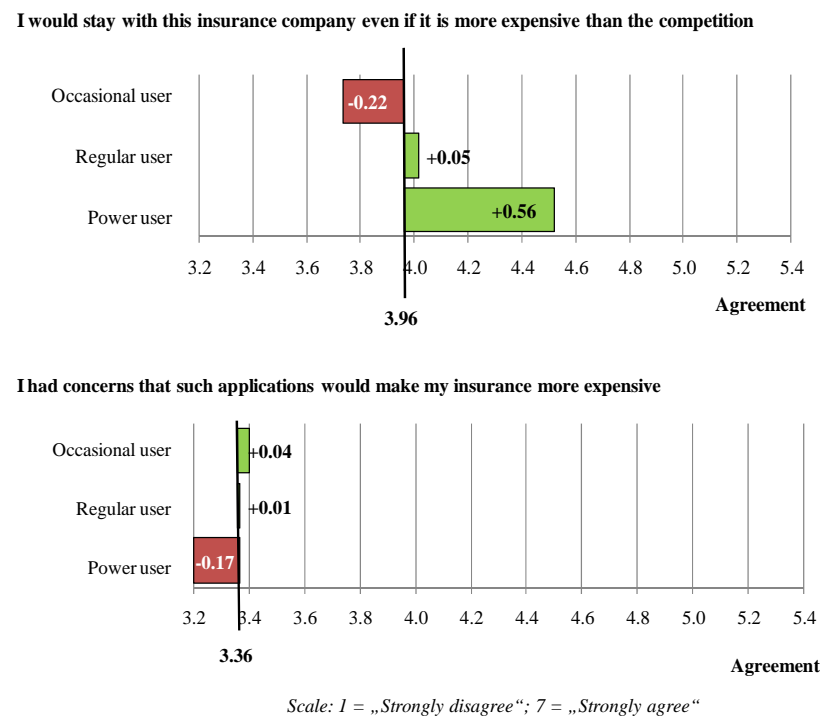

Figure 11. Willingness-to-Pay-More (by User Type) 
Similar differences between user types can be identified for the two items of the "willingness-to-pay-more" construct (see Figure 11). "Power users" regard themselves as more likely to stay with a slightly more expensive insurance company, if it offers mobile applications. However, the second measure of the construct indicates that mobile applications are not a means of creating additional revenue: Taking into account that a lot of mobile applications are free of charge, the possibilities for insurance companies to charge customers for mobile insurance applications are limited. Therefore, from a provider perspective, positive effects of mobile insurance applications have to be seen in their possibility to create positive word-of-mouth effects and, in case of the proposed claims assistance application, in reducing loss expenses. From a customer perspective, applications that provide help in the "moment-of-truth", i.e. a concrete emergency situation, have to be regarded as attractive and promising.

\section{DISCUSSION}

Our prototypical implementation of the proposed mobile claims assistance application achieved two main objectives: Firstly, we demonstrated the benefits of such an application for the different parties involved in an emergency situation such as a car accident. Secondly, we demonstrated that mobile phones are technically capable of reporting claims into commercial claims management enterprise systems as well as offering mobile Web Services to enable an asynchronous communication flow.

In this paper, we discussed an implementation of a mobile claims assistance application based on three different mobile phone platforms (Android, Blackberry OS, iPhone OS) and a claims management product (SAP Claims Management 6.0 EHP4). However, our prototype is not limited to the aforementioned platforms and products. The concept of Business Connectors and the Tunnel Architecture are platform-independent and allow for an easy integration of enterprise systems offered by various vendors, while the adherence to Web Service standards simplifies the integration of heterogeneous mobile phone platforms.

Of course, from a usability perspective, a mobile application is not suited to deal with all the questions one might have in the direct aftermath of a car accident. This also means that the submitted case circumstances are not sufficient to file a complete loss report, since car accidents require more complex case circumstances including for example a police report or evidences from witnesses. However, it is worth mentioning that it is not the intention of the proposed mobile claims assistance application to replace the personal contact to an emergency call center agent after a car accident, but to enable it at an earlier point in time and to support people with the loss report as well as by means of location-based services. Additionally, the application is designed with the goal of high usability taking into consideration the exceptional circumstances of a car accident rather than guaranteeing a comprehensive loss report. As studies concerning the claims management process show [8], it is more important for the insurance carrier to be notified about an accident as soon as possible than to receive the complete case circumstances right away. Initially submitted information comprises the current time and location of the accident as well as data about the persons involved, while additional information can be easily collected in the days after the incident in order to complete the loss report.
From a security perspective, the transmission of personal data and the authentication of the client devices are the main issues. Since our components are using WS-* standards, an additional encryption such as SSL/TLS could be applied. Also, mobile devices are using a low bandwidth connection compared to enterprise systems. Thus, a gzip compression as specified in the HTTP/1.1 protocol can be considered. For device authentication we used the IMSI number of the device, which is also used to identify a user within the provider network for incoming calls or text messages.

\section{CONCLUSIONS AND FUTURE WORK}

In this research paper, we proposed an application that assists people in the aftermath of car accidents based on mobile phones. Our prototypical implementation demonstrates how people can use mobile phones to submit an emergency call and report an insurance claim to a commercial claims management enterprise system, how they benefit from location-based value-added services, and how mobile Web Services enable insurance companies to request a customer feedback after a claim is settled. Based on the concepts for mobile claims assistance, we discussed the technical challenges that come along with a Web Service-based integration of mobile phones with enterprise systems and demonstrated how our solution addresses them.

In addition to the technical contribution, we provided results from several studies on the user perspective on mobile insurance that support our assessment that a mobile claims assistance application is of high relevance. The quantitative results from our second web-based empirical study amongst 2,000 participants indicate a high perceived usefulness and intention to use from a user perspective, while qualitative results from the focus groups show that insurance experts expect that the proposed solution can lead to savings in processing time and costs for insurance companies.

As future work, we plan to conduct experiments with a group of potential end users. As part of the experiments, participants will experience a simulated car accident and will use their mobile devices for claims assistance. The goal is to evaluate whether our prototype meets actual user needs after car accidents and to assess the usability of the application. From a technology perspective, further scalability tests are necessary, especially with respect to the transmission of images and videos of the accident scene. Another interesting area of research is the interaction with real world objects when reporting an accident. As an example, mobile phones in combination with Bluetooth technology could be used to retrieve crash data from the on-board unit of a car. Another example is the identification of a damaged car or even single components based on technologies such as barcodes or NFC.

Finally, future research should investigate which convenience services (e.g., the navigation to the cheapest close-by gas station) could complement the functional range of the proposed claims assistance application. Statistically a car accident occurs only every seven years, while the exchange rate of mobile phones is much higher. Therefore, it seems reasonable to combine the lowfrequency claims assistance application with high-frequency services in order to enable a faster market penetration of the application. 


\section{REFERENCES}

[1] Apple Push Notification Service Programming Guide: What Are Push Notifications?

http://developer.apple.com/iPhone/library/documentation/Net workingInter-

net/Conceptual/RemoteNotificationsPG/WhatAreRemoteNot if/WhatAreRemoteNotif.html

[2] Bottazzi, D., Corradi, A., and Montanari, R. 2006. Contextaware middleware solutions for anytime and anywhere emergency assistance to elderly people. IEEE Communications Magazine, 44, 4, 82-90.

[3] Coroama, V. and Langheinrich, M. 2006. Personalized vehicle insurance rates - a case for client-side personalization in ubiquitous computing. In Workshop on Privacy-Enhanced Personalization at CHI 2006.

[4] CreativeCoefficient. Crash Report. http://www.creativecoefficient.com/cr/

[5] Davis, F. D. 1989. Perceived Usefulness, Perceived Ease of Use, and User Acceptance of Information Technology. MIS Quarterly, 13, 3, 319-340.

[6] European Commission. eCall Toolbox. http://www.esafetysupport.org/en/ecall_toolbox/

[7] Festag, A., Baldessari, R., Zhang, W., Le, L., Sarma, A., and Fukukawa, M. 2008. CAR-2-X Communication for Safety and Infotainment in Europe. NEC Technical Journal, 3, 1.

[8] Guyan, V. and Hollander, D. 2002. Unlocking the Value in Claims. Accenture - Insurance Solution Group.

[9] ISO/IEC 18004. 2006. Information technology - Automatic identification and data capture techniques - QR Code 2005 bar code symbology specification.

[10] InventiveTouch Software Inc. C.A.R. Car Accident Report. http://www.inventivetouch.com/products/car/

[11] Jammes, F., Mensch, A., and Smit, H. 2007. ServiceOriented Device Communications Using the Devices Profile for Web services. In Proc. of AINA 2007, 947-955.

[12] Jolliffe, I. T. 2002. Principal Component Analysis. Springer Series in Statistics.

[13] Landgren, J. and Nulden, U. 2007. A study of emergency response work: patterns of mobile phone interaction. In Proc. of the SIGCHI conference on Human factors in computing systems (CHI’07), 1323-1332.

[14] Landry, B., Pierce, J., and Isbell, C. 2004. Supporting routine decision-making with a next-generation alarm clock. Personal and Ubiquitous Computing, 8, 3-4, Springer, 154-160.

[15] Ling R. 2004. The mobile connection: the cell phone's impact on society, Morgan Kaufmann, San Francisco, USA.

[16] Litman, T. 2008. Distance-Based Vehicle Insurance - Feasibility, Costs and Benefits. Comprehensive Technical Report, Victoria Transport Policy Institute.

[17] Meier, R. 2009. Professional Android Application Development. Wiley Publishing, Inc. USA.

[18] Nationwide. New App from Nationwide Insurance Helps iPhone Users After an Accident. Media release. http://www.nationwide.com/newsroom/nw-iPhone-app.jsp

[19] Nysveen, H., Pedersen, P. E., and Thorbjørnsen, H. 2005. Intentions to use mobile services: Antecedents and crossservice comparisons. Journal of the Academy of Marketing Science, 33, 3, 330-346.

[20] OASIS 2009. Devices Profile for Web Services Version 1.1. http://docs.oasis-open.org/ws-dd/dpws/1.1/os/wsdd-dpws1.1-spec-os.pdf.

[21] Octo Telematics. Insurance Telematics. http://www.octotelematics.com/solutions/insurancetelematics/

[22] Schall, D., Aiello, M., and Dustdar, S. 2006. Web Services on Embedded Devices. International Journal of Web Information Systems, 2, 1, 45-50.

[23] Signer, B., Norrie, M., Geissbuehler, P., and Heiniger, D. 2002. Telephone Interface for Avalanche Warnings based on Information Server for Adaptable Content Delivery. In Proc. of the International Conference on Pervasive Computing, 725-740.

[24] Zeithaml, V. A., Berry, L. L., and Parasuraman, A. 1993. The Nature and Determinants of Customer Expectations of Service. Journal of the Academy of Marketing Science, 21, 1, 112.

[25] Zeithaml, V. A., Berry, L. L., and Parasuraman, A. 1996. The Behavioral Consequences of Service Quality. Journal of Marketing, 60, 2, 31-46. 